\title{
Osmolality as a Measure of Dehydration in the Neonatal Period
}

\author{
J. A. DAVIS, D. R. HARVEY, and J. F. STEVENS \\ From the Nuffield Neonatal Research Unit, Hammersmith Hospital, London W.12
}

True dehydration implies an increase in the osmolality of the extracellular fluid, of which the plasma provides a modified sample, and is conventionally measured by estimating the plasma sodium level which in most circumstances represents half the plasma osmolality (sodium in millinormals and osmolality in milliosmoles/kg. water); however, this relationship does not always hold, for instance in the dying (Rubin, Braveman, Dexter, Vanamee, and Roberts, 1956). It is known that there is a physiological mechanism for the control of the concentration of the extracellular fluid which is geared to total osmolality (Verney, 1946) and is independent of the control of the concentrations of individual solutes (e.g. sodium ions). Plasma sodium levels are very variable immediately after birth (Acharya and Payne, 1965), but hitherto it has not been shown whether these changes reflect equivalent alterations in plasma osmolality. Direct measurement of osmolality should settle this point and provide a more accurate assessment than sodium levels of hydration and dehydration in the newborn. It is of course normal for an infant to lose some weight in the first few days of life, the loss being made up by about the tenth day, and J. Puga and J. P. M. Tizard (1965, personal communication) have shown that this loss amounts to $40-50 \mathrm{~g} . / \mathrm{kg}$. day for the first 2 days of life in the full-term baby. About half this loss is insensible, and it is reasonable to postulate that it will represent loss of water rather than of isotonic fluid, and should, therefore, be accompanied by a rise in the osmolality of the extracellular fluid. It is part of the folklore of paediatrics that fever at this time of life may be the result of dehydration, which presumably limits the ability of the infant to keep cool in a warm environment, and in that case it should be accompanied by an excessive rise in plasma osmolality rather than by signs of infection. For these reasons, and because both in practice and from a theoretical point of view knowledge of the

Received January 5, 1965. degree of dehydration may be important, it was decided to carry out serial estimations of plasma osmolality in a series of newborn infants, from birth till the end of the first week of life, and the establishment of a satisfactory feeding régime.

\section{Material and Methods}

Two classes of newborn infant were studied.

(i) Normal full-term healthy infants in the lying-in wards. The purpose and nature of the investigation was explained to the mothers of the babies concerned, who almost without exception allowed us to take daily blood samples. Samples were collected by heel-prick without undue squeezing, and no precautions against loss of $\mathrm{CO}_{2}$ were taken. All haemolysed samples were discarded. Samples at birth were obtained from the umbilical cord. Estimations were carried out within 6 hours of sampling in all instances. The feeding régime of these infants was not uniform; some being breast-fed on demand, others bottle-fed by the clock; but care was taken always to collect samples at 9 a.m., just before the next feed was due.

(ii) Previously healthy, full-term babies in the lying-in wards who were found to be feverish; fever being defined as a temperature of $37.5^{\circ} \mathrm{C}$. on two successive routine observations. Rectal temperatures were taken by the nursing staff using mercury thermometers inserted for one minute and were always checked when found to be raised. During the five-month period of the study, 10 babies fulfilled these criteria, and of these, 6 were studied. These babies showed an average weight loss of $70 \mathrm{~g} . / \mathrm{kg}$. $24 \mathrm{hr}$.

Osmolality was measured cryoscopically using the Fiske osmometer.

Sodium was measured by the method of Stevens (1965) using an E.E.L. flame photometer. The same samples were used for both estimations. Capillary blood was collected into heparinized tubes and the estimations were carried out as soon as possible after collection and in every case within 6 hours. Duplicate readings on the Fiske osmometer were found to agree within $1 \%$, and serial readings on given samples showed only the same degree of variation when made 120 hours apart.

Heparin was only used in the minimal quantities needed to prevent clotting. The amount of sodium present did not raise the estimated level by more than $1 \mathrm{mEq} / \mathrm{l}$. 


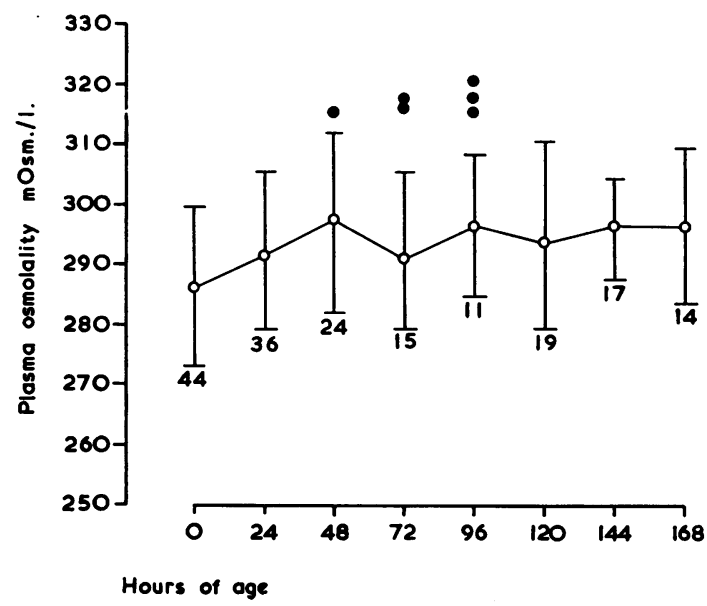

FIG. 1.-The mean plasma osmolality with two standard deviations during the first week of life in normal and dehydrated infants. There is a general rise in the first 48 hours similar to that previously described by Gautier (1964), with a levelling-off thereafter.

$\circ=$ mean normal $( \pm 2 S D) ; \bullet=$ dehydrated.

(The number of estimations on each day is also shown.)

\section{Results}

The results are given in Fig. 1-3. On each of the figures we have superimposed the levels of the 6 feverish infants who were studied. In every case the plasma osmolality deviates by more than two standard deviations from the mean for normal infants of similar age from birth, whereas the sodium

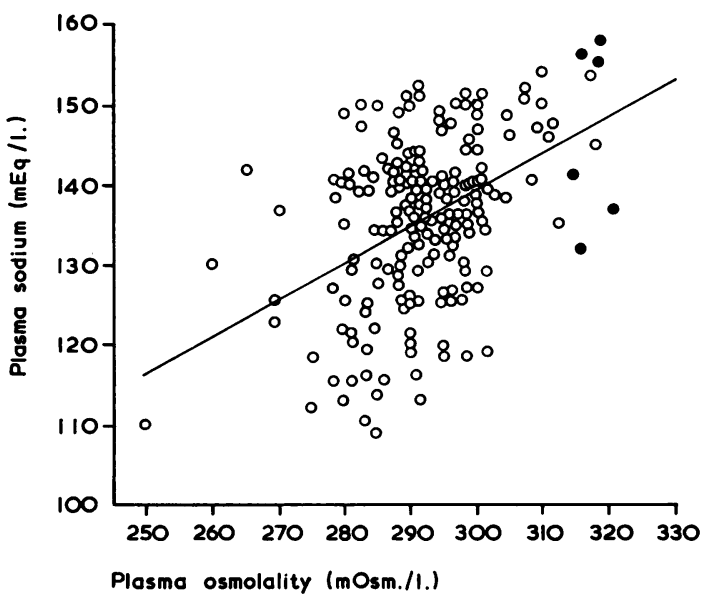

FIG. 3.-Correlation between sodium and osmolality levels in normal and dehydrated babies.

$\circ=$ normal $; \bullet=$ dehydrated .

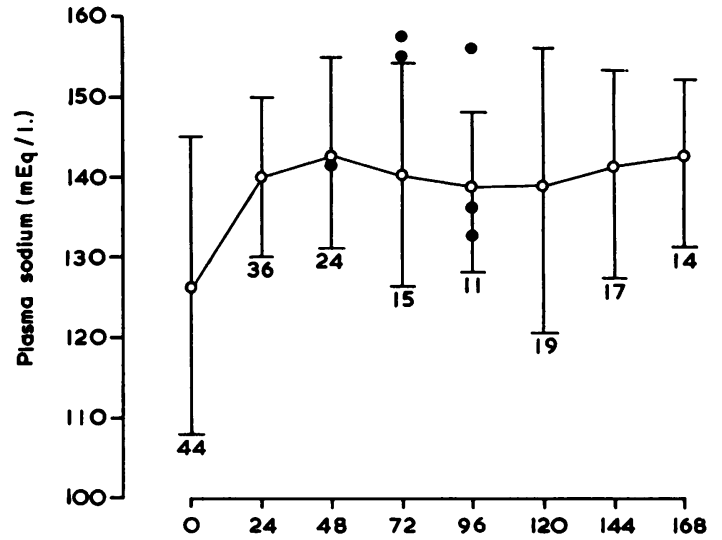

Hours of age

FIG. 2.-Plasma sodium levels in normal and dehydrated babies.

$\circ=$ mean normal $( \pm 2 S D) ; \bullet=$ dehydrated. (The number of estimations on each day is also shown.)

levels in 3 out of the 6 babies fell well within the normal range.

\section{Discussion}

The data show clearly that plasma osmolality and serum sodium bear no fixed relationship to each other during the first week of life (correl. coeff. = $0.458(p=0 \cdot 15))$. Therefore, in this period of life sodium levels are not an indirect measure of osmolality, as they are in the normal subject. Since all 6 of the babies who developed significant fever during the period of study and were investigated also showed a significantly raised plasma osmolality, it is reasonable to conclude that fever and dehydration are closely associated in the newborn infant; and since none of these infants were ill or showed signs of infection, and all recovered with no more treatment than the giving of extra fluids and the provision of a cooler environment, it is likely that the dehydration was the cause of the fever rather than vice versa -thus supporting the views of Craig (1963) who believes that fever in the newborn period should not be regarded as necessarily due to infection. In fact, during the period of study we encountered 2 infants with serious infection, and of these one showed a very large rise in plasma osmolality, whereas in the other the level was within the normal range. The presence of a raised osmolality does not, therefore, exclude infection, but fever in the presence of a normal osmolality makes infection very likely. We conclude that in assessing dehydration in sick infants and the need for parenteral fluid therapy the 
plasma osmolality should be estimated in preference to the sodium level, and that in any assessment of renal and posterior pituitary function in the newborn the plasma osmolality level should be used as the guide to the stress imposed.

Since we have only measured the plasma sodium level, we do not know which substances cause the increased osmolality. It is interesting to note that Rubin et al. (1956), who determined the plasma osmolality in dying patients, were unable to account for this rise by calculation from the measured components of plasma.

\section{Summary}

Plasma osmolality and sodium levels were estimated at birth and daily thereafter in a number of healthy full-term infants and in a series of feverish infants.

There was only a slight correlation between serum sodium and plasma osmolality readings.

A series of feverish babies without signs of infection showed a significant rise in osmolality but not in sodium levels.

Implications are discussed.

We should like to thank Sister Day for her help and Miss D. Norman for performing the estimations. This work was supported by grants from the Sir William Coxen Trust and the Nuffield Foundation.

\section{REFERENCES}

Acharya, P. T., and Payne, W. W. (1965). Blood chemistry of normal full-term infants in the first 48 hours of life. Arch. Dis. Childh., 40, 430.

Craig, W. S. (1963). The early detection of pyrexia in the newborn. ibid., 38, 29.

Gautier, E. (1964). Neonatal hyperosmolality, an instance of unresponsiveness to antidiuretic hormone. In Nutricia Symposium on the Adaptation of the Newborn Infant to Extrauterine Life, ed. J. H. P. Jonxis, H. K. A. Visser, and J. A. Troelstra, p. 83. H. E. Stenfert Kroese, Leyden.

Rubin, A. L., Braveman, W. S., Dexter, R. L., Vanamee, P., and Roberts, K. E. (1956). The relationship between plasma osmolality and concentration in disease states. Clin. Res. Proc., 4, 129.

Stevens, J. F. (1965). Biochemical changes in the blood of the newborn. f. med. lab. Tech., 22, 47.

Verney, E. B. (1946). Absorption and excretion of water; the antidiuretic hormone. Lancet, 2, 739 and 781.

\section{Notice}

The IVth International Symposium on Cystic Fibrosis of the Pancreas (Mucoviscidosis) and second administrative session of the International Cystic Fibrosis (Mucoviscidosis) Association will be held in Berne, September 19-22, 1966.

\section{Programme:}

1. Chemistry of the glucoprotein and mucous secretion.

2. Serous secretion.

3. Clinical investigations.

4. Genetics.

5. Therapy.

6. Social aspects.

Information can be obtained from Dr. B. Friolet, Universitäts-Kinderklinik (Prof. E. Rossi) 3008 Berne, Switzerland. 